\title{
The study of quantitative optical absorption imaging by using Monte Carlo simulation of combined photoacoustic tomography and ultrasound-modulated optical tomography
}

Chulhong Kim, Yang Li, Lihong V. Wang

Chulhong Kim, Yang Li, Lihong V. Wang, "The study of quantitative optical absorption imaging by using Monte Carlo simulation of combined photoacoustic tomography and ultrasound-modulated optical tomography," Proc. SPIE 8223, Photons Plus Ultrasound: Imaging and Sensing 2012, 82232C (23 February 2012); doi: 10.1117/12.905792

SPIE. Event: SPIE BiOS, 2012, San Francisco, California, United States 


\title{
The study of quantitative optical absorption imaging by using Monte Carlo simulation of combined photoacoustic tomography and ultrasound-modulated optical tomography
}

\author{
Chulhong $\mathrm{Kim}^{1 *}$, Yang $\mathrm{Li}^{1}$, and Lihong V. Wang ${ }^{2}$ \\ ${ }^{1}$ Bio optics and acoustics Laboratory, Department of Biomedical Engineering, The State University \\ of New York at Buffalo, 332 Bonner Hall, Buffalo, New York, 14260 \\ ${ }^{2}$ Optical Imaging Laboratory, Department of Biomedical Engineering, Washington University in St. \\ Louis, Campus Box 1097, One Brookings Dr. St. Louis, Missouri, 63130 \\ *Corresponding author: chulhong@buffalo.edu
}

\begin{abstract}
We demonstrate the feasibility of quantitative optical absorption imaging by using Monte Carlo simulation of combined photoacoustic tomography (PAT) and ultrasound-modulated optical tomography (UOT). Our simulation results show that the optical fluence on initial photoacoustic (PA) pressure waves can be accurately compensated for recovering exact optical absorption coefficients by using a fluence map acquired from UOT signals. Further, when the optical heterogeneities of a sample were varied, the recovered optical absorption coefficients from a target remained constant while PA amplitudes fluctuated. In practice, PAT and UOT systems can be potentially combined because both imaging systems can easily share light illumination and ultrasound application patterns.
\end{abstract}

Keywords: Photoacoustic tomography, ultrasound-modulated optical tomography, oxygen saturation of hemoglobin, optical absorption coefficients, Monte Carlo simulation.

\section{INTRODUCTION}

Optical imaging has great promise as an imaging modality due to its nonionizing radiation, low cost, portability, and high sensitivity. ${ }^{1}$ However, owing to strong light scattering, conventional optical imaging techniques suffer from either shallow penetration (e.g., optical microscopy ${ }^{2}$ ) or poor spatial resolution (e.g., diffuse optical tomography (DOT) $)^{3}$ ). Two types of ultrasound-mediated optical tomography - photoacoustic tomography (PAT) and ultrasound-modulated optical tomography (UOT) - are hybrid biomedical imaging techniques that overcome the drawbacks of conventional optical imaging modalities. ${ }^{4,5}$ They can provide rich optical contrast with high ultrasonic resolution, based on internally generated ultrasound caused by short laser pulse irradiation in PAT or externally applied ultrasound in UOT. The spatial resolution and imaging depth of both techniques are scalable with ultrasonic frequency. Therefore, these imaging modalities break through the fundamental limitations of pure optical imaging in penetration depth while maintaining high spatial resolution.

PAT has recently gained great attention because it clearly delineates morphological, functional (e.g., total hemoglobin concentration and hemoglobin oxygen saturation $\left(\mathrm{SO}_{2}\right)$ ), and molecular information of biological tissues in vivo, based on optical absorption properties. ${ }^{1,4,6}$ In PAT, a short-pulsed laser is used to irradiate the tissue, and photoacoustic (PA) waves are excited via thermoelastic expansion. The generated PA waves propagate in the medium and are then detected by an ultrasonic transducer. The initial PA pressure rises $\left(P_{0}\right)$ are proportional to the product of local optical energy deposition $\left(\mu_{a} F\right)$ and other thermal and mechanical properties $(\Gamma)$, where $\mu_{a}$ denotes the optical absorption coefficient of

Photons Plus Ultrasound: Imaging and Sensing 2012, edited by Alexander A. Oraevsky, Lihong V. Wang, Proc. of SPIE Vol. 8223, 82232C · C 2012 SPIE · CCC code: 1605-7422/12/\$18 · doi: 10.1117/12.905792 
a target, $F$ denotes local optical fluence, and $\Gamma$ is the Gruneisen parameter. ${ }^{4}$ The acquired PA data, based on the arrival times of the ultrasonic waves, are then used to quantify the optical absorption distribution. PAT image contrast is determined by optical absorption in the PA excitation phase, whereas its resolution is derived from ultrasonic detection in the PA emission phase. Most importantly, the amplitudes of PA waves are directly proportional to the optical absorption coefficients of targets.

UOT is another type of ultrasound-mediated optical tomography. Like PAT, it offers strong optical contrast with high ultrasonic spatial resolution. ${ }^{1,5}$ In UOT, high-coherence light illuminates biological tissues that are insonified with ultrasound. Light traveling through the ultrasonic focal spot is ultrasonically modulated so that each speckle grain at a detector surface has a time-varying modulation component due to the acousto-optic interaction. By measuring the degree of modulation at each scanned ultrasonic focal spot, the optical properties as well as acoustic properties of the tissue are imaged. Since any light (scattered or unscattered) modulated by ultrasonic interactions contributes to UOT signals, the imaging depth can be extended beyond one optical transport mean free path $(\sim 1 \mathrm{~mm})$. Because UOT uses localized ultrasonic modulation of light, it can provide higher spatial resolution than DOT while offering the same optical contrast (local optical fluence including both absorption and scattering). Compared to PAT, UOT is capable of supplying optical scattering and mechanical properties as well as optical absorption properties, whereas PAT is mostly sensitive to optical absorption. UOT images are not as sharp as PAT images because UOT signals are proportional to local optical fluence, while PA signals are proportional to the product of the local absorption coefficient and local optical fluence. However, there is no rejection of un-resolvable background signals in UOT images (which is also true for DOT). Moreover, because the image contrast of UOT is based on optical properties, this technique is potentially able to supply morphological and functional information about tissues. ${ }^{7,8}$

Quantitative measurement of optical absorber concentration is extremely important for accurate imaging of in vivo physiological functions such as tumor hyper-metabolism and brain functions. By tuning optical wavelengths, spectroscopic PAT (sPAT) has been applied to quantitatively measure the concentration of various intrinsic chromophores (e.g., water, oxyhemoglobin, deoxyhemoglobin, lipid, and melanin). However, local optical fluence varies widely at different optical wavelengths, and consequently the SPAT itself is not suitable for measuring accurate concentrations of multiple chromophores. To compensate for spectral variation of local optical fluence, many methods

have been explored. ${ }^{9-11}$ A correction factor acquired from ex vivo measurement has been applied to compensate for the spectral variation of light attenuation during in vivo imaging. Mathematical models or introduction of exogenous contrast agents have also been applied to extract the chromophore distribution from the measured PA signals. However, due to the high complexity and heterogeneity of biological tissues, all these methods have limited accuracy for quantitative PAT. Therefore, a non-invasive general method is needed.

Quantitative imaging of absorber concentrations requires a map of $\mu_{a}$. PAT, however, reconstructs $P_{0}$, directly. Since $P_{0}$ is proportional to $\mu_{a} F, F$ is needed to recover $\mu_{a}$ through $\mu_{a} \propto P_{o} / F$. Note that both $F$ and its normal gradient across tissue types are continuous since the bulk averaged refractive index is relatively uniform in tissue. As a result, $F$ is spatially smooth and slowly varying, and low-resolution DOT has been applied to compensate for $F$ in quantitative PAT. ${ }^{12}$ However, the relatively higher-resolution of $F$ distribution will enhance the accuracy in quantitative PAT, so UOT is a perfect candidate for this purpose.

In this proceedings, we show the feasibility of quantitative mapping of optical absorption coefficients by using a Monte Carlo (MC) simulation of combined photoacoustic and ultrasound-modulated optical tomography. Our simulation results prove that the fluence map obtained from UOT accurately compensates for the optical fluence on initial PA pressure waves and permits recovery of exact optical absorption coefficients. Further, although the optical heterogeneities of the sample varied, the optical absorption coefficients from a target, recovered by using the ratio of between PAT and UOT signals, remained constant, whereas PA amplitudes fluctuated. Practically speaking, these two ultrasound-aided optical tomography systems can easily be integrated.

\section{METHODS AND MATERIALS}

To investigate the feasibility of using a combined PAT and UOT system for recovery of optical absorption coefficients $\left(\mu_{a}\right)$, we used a MC method to simulate distributions of initial PA pressure waves and ultrasound-modulated light intensities in an optically scattering medium including optically absorptive objects. Then, the ratio between the two 
signals was used to recover the original $\mu_{a}$ of the objects. The MC simulation is based on the temporal correlation transfer equation for acoustically-modulated, multiply-scattered light. ${ }^{13}$ Using this method, we could map the distribution of ultrasound-modulated light intensity and optical fluence $(F)$ in an isotropic optical scattering medium (i.e., scattering anisotropy $=0$ ) with heterogeneous optical properties and a nonuniform ultrasonic field. The simulation parameters included a cylindrical ultrasonic focal zone $2 \mathrm{~mm}$ in diameter and $20 \mathrm{~mm}$ in length, an ultrasonic pressure of 1.5 MPa, an ultrasonic frequency of $1 \mathrm{MHz}$, and an optical wavelength of $632 \mathrm{~nm}$. One hundred millions photons were used for each simulation. The simulation schematic is shown in Fig. 1. A pencil beam was normally incident along the $Z$ axis on a $10 \times 10 \times 2 \mathrm{~cm}$ (along the $\mathrm{X}, \mathrm{Y}$, and $\mathrm{Z}$ axes, respectively) scattering medium with a reduced scattering coefficient of $10 \mathrm{~cm}^{-1}$ and a background absorption coefficient of $0.1 \mathrm{~cm}^{-1}$. Three optically absorptive objects were embedded in the scattering medium. Objects 1 and 2, measuring $2 \times 2 \times 2 \mathrm{~mm}$ (along the $\mathrm{X}, \mathrm{Y}$, and $\mathrm{Z}$ axes, respectively) and with a $\mu_{a}$ of $6 \mathrm{~cm}^{-1}$, were positioned in the middle plane. The center to center distance between these two objects was $8 \mathrm{~mm}$ along the $\mathrm{X}$ axis. Then, Object 3 , measuring $4 \times 2 \times 2 \mathrm{~mm}$ (along the $\mathrm{X}, \mathrm{Y}$, and $\mathrm{Z}$ axes, respectively) and with a $\mu_{a}$ of $10 \mathrm{~cm}^{-1}$, was included $2.5 \mathrm{~mm}$ distant along the $\mathrm{Z}$ axis from the center of Object 1 . Our hypothesis was that the $F$ around Object 1 would be significantly disturbed by Object 3 , whereas this disturbance around Object 2 would be less significant. Ultrasound waves were applied against the $\mathrm{X}$ axis. We mapped the distribution of initial PA pressure waves in the medium by multiplying the known $\mu_{a}$ map with the $F$ map acquired from the MC simulation. In a practical PAT system, the propagated PA waves are detected by an ultrasound transducer. However, we ignored acoustic heterogeneities of the medium in this simulation. The recovery process of optical absorption coefficients included the following steps: (1) form high-resolution $P_{0}$ images using PAT, (2) form lowresolution $F$ maps using UOT, and (3) compute high-resolution $\mu_{a}$ maps using $\mu_{a} \propto P_{o} / F$.

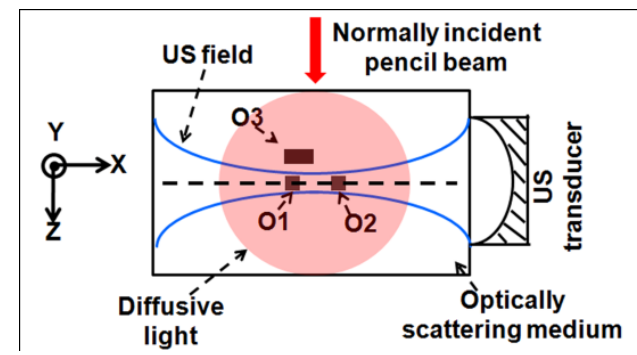

Figure 1: Schematic of Monte Carlo simulation. US, ultrasound and O, objects.

\section{RESULTS AND DISCUSSION}

First, to prove the feasibility of the use of ultrasound-modulated light intensity (UOT signals) as a fluence $(F)$ compensating factor in PAT images, we compared two light distributions of UOT signals and local $F$ in Fig. 2. These two 1-D profiles were taken along the dotted line in Fig. 1, and two dips in the profiles corresponded to the positions of Objects 1 and 2. Although the absolute light intensities of $F$ are $\sim 33$ times stronger than the UOT signals (Fig. 2a), the normalized light intensities of the two profiles are nearly identical (Fig. 2b). Low signal-to-noise ratios (SNR) in UOT are well known, and are due to uncorrelated speckle grains and low light-ultrasound interaction efficiency. Nevertheless, here the UOT signals can be clearly detected using current UOT systems without any mathematical reconstruction algorithm, yet the local $F$ cannot be clearly mapped using any current imaging technique.
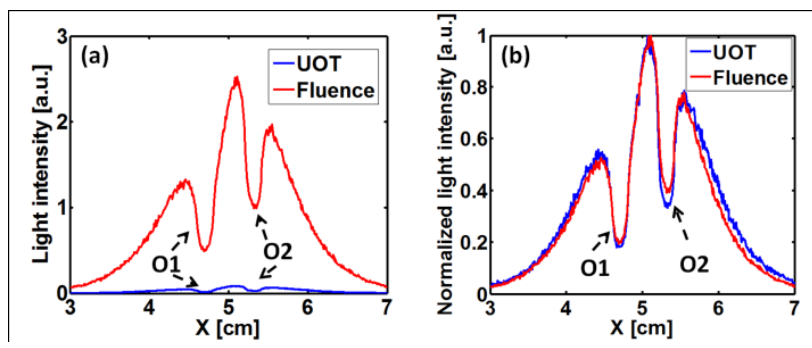

Figure 2: Comparison of 1-D profiles of ultrasound-modulated light intensity and optical fluence, cut along the dotted line in Fig. 1. (a) Absolute light intensity and (b) normalized light intensity. O, objects. 
Next, we obtained the recovered $\mu_{a}$ of Objects 1 and 2 (O1 and $\mathrm{O} 2$ in Fig. 1.) by taking the ratio between the PAT and UOT signals in Fig. 3. Figure 3a shows a 1-D profile of initial PA pressure waves, a 1-D depth-resolved image (A-line) which can be acquired by a practical PAT system, cut along the dotted line in Fig. 1. We can clearly see that the PA signal from Object 1 is lower than that from Object 2 although the $\mu_{a}$ of both objects are identical. A 1-D profile of UOT signals, an A-line image which can be obtained by a practical UOT system, is shown in Fig. 3b. The decrease in UOT signals within Object 1 is more than that of Object 2 although the $\mu_{a}$ of both objects are identical. Thus, we could conclude that the existence of Object 3 quantitatively disturbed the measurements in both PAT and UOT images, and neither of them could independently map the true $\mu_{a}$ of the objects. However, the ratio between the two A-lines (i.e., the PAT A-line divided by the UOT one) clearly recovered the $\mu_{a}$ of Object 1 (Fig. 3c), and matches well with the true $\mu_{a}$ of both objects (Fig. 3d). Note that the recovered $\mu_{a}$ is not absolute but relative. However, we can recover the absolute $\mu_{a}$ by calibrating a PAT/UOT system with a tissue phantom of known $\mu_{a}$.

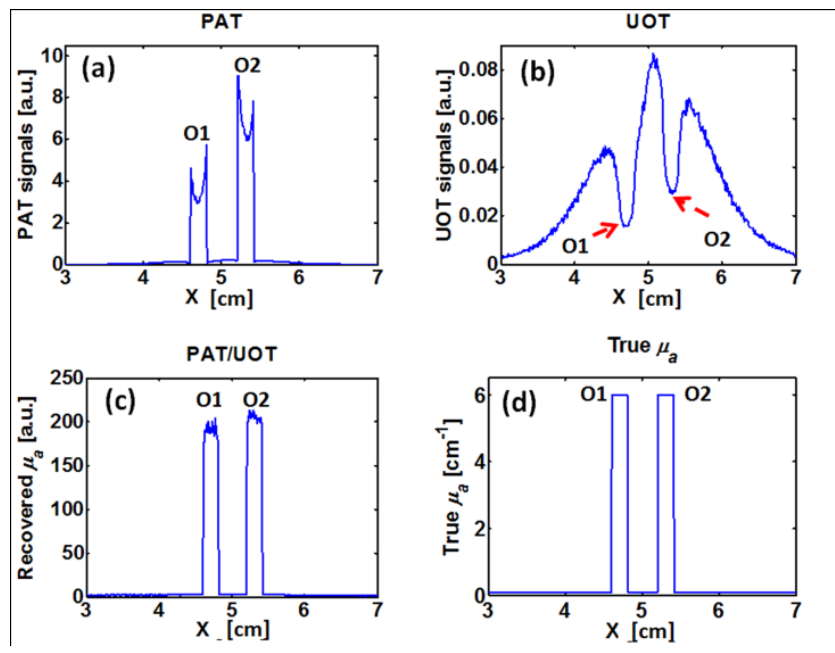

Figure 3: Monte Carlo simulation results of recovered optical absorption coefficients $\left(\mu_{a}\right)$. (a) PAT A-line image cut along the dotted line in Fig. 1. (b) UOT A-line image. (c) Recovered $\mu_{a}$ by taking the ratio between PAT and UOT A-line images. (d) True $\mu_{a}$. O, objects.

In further simulations, we varied the $\mu_{a}$ of Objects 1 and 2 from 1 to $10 \mathrm{~cm}^{-1}$, while simultaneously varying Object 3's $\mu_{a}$ from 2.5 to $10 \mathrm{~cm}^{-1}$ (inset legend in Fig. 4). From Fig. 4, three observations are apparent: (1) The ratio of the PAT to the UOT signals measured from Objects 1 (Fig. 4a) and 2 (Fig. 4c) is linearly proportional to the preset $\mu_{a}$ of Objects 1 and 2. (2) The recovered $\mu_{a}$ at a given preset $\mu_{a}$ of Objects 1 and 2 remains constant despite varying the $\mu_{a}$ of Object 3. (3) Although the local $F$ of Object 1 is more affected by Object 3 than that of Object 2, the recovered $\mu_{a}$ values between the two objects are identical. Three further observations are that (1) PAT signals do not increase linearly with the preset $\mu_{a}$ of Objects 1 and 2. (2) They are significantly disturbed by changes in the $\mu_{a}$ of Object 3. At a given preset $\mu_{a}$ of Objects 1 and 2, the calculated PAT signals differ with varying $\mu_{a}$ for Object 3 . This discrepancy is larger for Object 1 than for Object 2, where the influence of Object 3 is more significant. For example, the PAT signals from Object 1 at a preset $\mu_{a}$ of $10 \mathrm{~cm}^{-1}$ changed by $45 \%$ when Object 3's $\mu_{a}$ varied from 2.5 to $10 \mathrm{~cm}^{-1}$. However, the PAT signals from Object 2 changed by only $6 \%$ with the same variation in Object 3's $\mu_{a}$. (3) Because the local $F$ of Object 1 was reduced by the existence of Object 3, the estimated PAT signals from Object 1 are less than those from Object 2. These MC simulation results prove that the ratio between two A-lines acquired by a PAT and a UOT system can easily recover the absolute $\mu_{a}$ of tissue without using any mathematical model or requiring an exogenous contrast agent. 

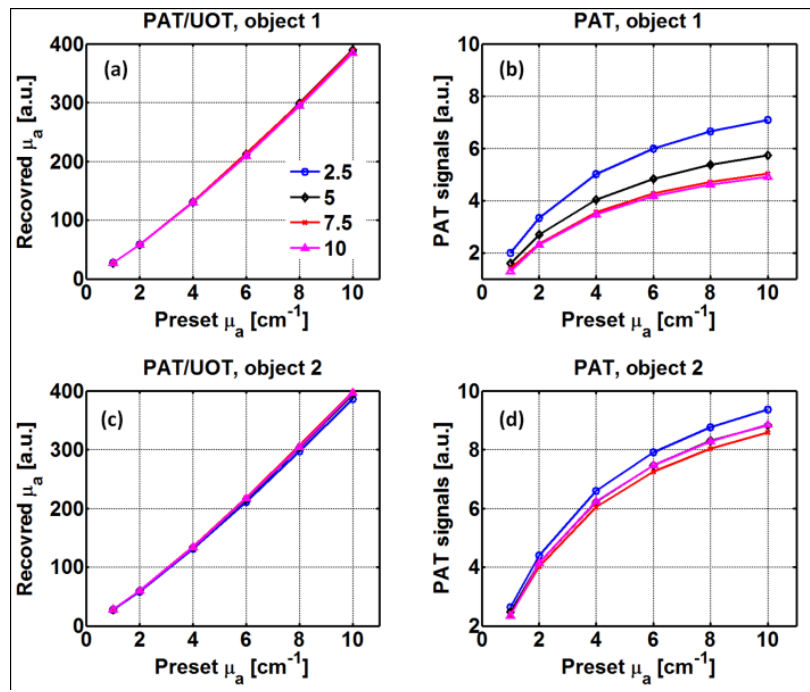

Figure 4: Recovered $\mu_{a}$ of Object 1 (a) and Object 2 (c), found by taking the ratio between PAT and UOT signals. PAT signals of Object 1 (b) and Object 2 (d). The $\mu_{a}$ of Objects 1 and 2 were varied from 1 to $10 \mathrm{~cm}^{-1}$, and the $\mu_{a}$ of Object 3 was varied from 2.5 to $10 \mathrm{~cm}^{-1}$. The legend in Fig. 4a shows the various $\mu_{a}$ of Object 3.

\section{CONCLUSIONS}

In conclusion, quantitative mapping of various optical absorber concentrations is crucial to the in vivo study of physiological functions such as tumor hyper-metabolism and brain functions. Our Monte Carlo simulation results successfully demonstrate that the optical absorption coefficients can be accurately recovered by using the ratio between PAT and UOT signals. This method requires no mathematical reconstruction algorithm or introduced contrast agent. The recovered $\mu_{a}$ using both PAT and UOT signals is consistently immune to complex optical heterogeneities. Song et. $a l .{ }^{14}$ and Kim et. $a l .{ }^{15}$ have independently built dark-field confocal PAT and UOT systems, respectively. Practically speaking, these two systems can easily be integrated without any major modification. Then, the practicability of the combined system can be greatly enhanced for in vivo imaging.

\section{ACKNOWLEDGEMENT}

This work was supported by the University at Buffalo Research Foundation faculty start-up fund.

\section{REFERENCES}

[1]. L.V. Wang and H. I. Wu, "Biomedical Optics: Principles and Imaging," (John Wiley \& Sons, Inc, Hoboken, NJ, 2007).

[2]. D. Huang, E. A. Swanson, C. P. Lin, J. S. Schuman, W. G. Stinson, W. Chang, M. R. Hee, T. Flotte, K. Gregory, C. A. Puliafito, and et al., "Optical coherence tomography," Science 254, 1178-1181 (1991).

[3]. B. W. Zeff, B. R. White, H. Dehghani, B. L. Schlaggar, and J. P. Culver, "Retinotopic mapping of adult human visual cortex with high-density diffuse optical tomography," Proc Natl Acad Sci U S A 104, 12169-12174 (2007).

[4]. C. Kim, C. Favazza, and L. V. Wang, "In vivo photoacoustic tomography of chemicals: high-resolution functional and molecular optical imaging at new depths," Chem Rev 110, 2756-2782 (2010). 
[5]. C. Kim, R. J. Zemp, and L. V. Wang, "Intense acoustic bursts as a signal-enhancement mechanism in ultrasoundmodulated optical tomography," Opt Lett 31, 2423-2425 (2006).

[6]. C. Kim, E. C. Cho, J. Chen, K. H. Song, L. Au, C. Favazza, Q. Zhang, C. M. Cobley, F. Gao, Y. Xia, and L. V. Wang, "In Vivo Molecular Photoacoustic Tomography of Melanomas Targeted by Bioconjugated Gold Nanocages," ACS Nano 4, 4559-4564 (2010).

[7]. C. Kim, and L. V. Wang, "Multi-optical-wavelength ultrasound-modulated optical tomography: a phantom study," Opt Lett 32, 2285-2287 (2007).

[8]. A. Bratchenia, R. Molenaar, T.G. van Leeuwen, and R. P. Kooyman, "Millimeter-resolution acousto-optic quantitative imaging in a tissue model system," J Biomed Opt 14, 034031 (2009).

[9]. X. Wang, X. Xie, G. Ku and L. V. Wang, "Noninvasive imaging of hemoglobin concentration and oxygenation in the rat brain using high-resolution photoacoustic tomography," J. Biomed. Opt. 11, 024015 (2006).

[10]. L. Yao, Y. Sun, and H. Jiang, "Quantitative photoacoustic tomography based on the radiative transfer equation," Opt Lett 34, 1765-1767 (2009).

[11]. J. R. Rajian, P.L. Carson, and X. Wang, "Quantitative photoacoustic measurement of tissue optical absorption spectrum aided by an optical contrast agent," Opt Express 17, 4879-4889 (2009).

[12]. A. Q. Bauer, R. E. Nothdurft, C. Li, L. V. Wang, and J. P. Culver, "Correcting for Heterogeneous Fluence Profiles in Photoacoustic Imaging with Diffuse Optical Tomography," in Biomedical Optics, OSA Technical Digest (CD) (Optical Society of America, 2010), paper BTuD24.

[13]. S. Sakadzic and L.V. Wang, "Correlation transfer equation for ultrasound-modulated multiply scattered light," Phys Rev E 74, 036618 1-10 (2006).

[14]. K. Song, and L. V. Wang, "Deep reflection-mode photoacoustic imaging of biological tissue," J Biomed Opt 12,060503 (2007).

[15]. C. Kim, K. H. Song, and L. V. Wang, "Sentinel lymph node detection ex vivo using ultrasound-modulated optical tomography," J Biomed Opt 13, 020507 (2008). 